\title{
Aircraft landing orbit design
}

\section{Zhang min}

School of Math and Physics, University of South China,

Hengyan, 4210012

Emin1217@sina. com

Huang zhen jiang

School of Math and Physics, University of South China,

Hengyan, 4210012

\begin{abstract}
The aircraft soft-landing trajectory design and control strategy of in-depth discussion and analysis of considering The aircraft landing during the motion, and the stress is different, a mathematical model, answers the questions posed by the topic. Considered actual flight situation, we established landing cabin mathematics model description The Aircraft, of movement State, according to established of model, repeatedly set angle of pitch by using Matlab , looking for meet design requirements optimal solutions, obtained optimal design points location of cross, and ordinate; in according to geometry obtained in recent months points and optimal design points of angle, according to longitude degrees calculation method, determines in recent months points, and far months points of location; When the aircraft landing elliptical orbit, it follows the conservation of energy, so the Aircraft, in recent months points and far months points of energy equal, and due to aircraft, in in recent months points and far months points of speed are and aircraft, to months heart of line vertical, is angular momentum conservation, according to gold proportions substitution, obtained has in recent months points and far months points of speed size and
\end{abstract} direction.

\author{
Liang zhan ping \\ School of Math and Physics, University of South China, \\ Hengyan, 4210012 \\ Tan jun \\ School of Math and Physics, University of South China, \\ Hengyan, 4210012
}

Key words: conservation of energy conservation of angular momentum of Newton's second law

\section{INTRODUCTION}

\subsection{Background}

aircraft successful launch of 1:30 on December 2, 2013, December 6, arrived in lunar orbit. aircraft in landing preparations in orbit quality to $2.4 \mathrm{t}$, install it on the lower part of the main reduction adjustable thrust engines capable of producing $1500 \mathrm{~N}$ to $7500 \mathrm{~N}$, its specific impulse (that is, the thrust per unit mass of propellant) to $2940 \mathrm{~m} / \mathrm{s}$ to meet the adjustment speed control requirements. Attitude adjustment motor is installed on all sides, given after the final direction of the thrust of the engine, can automatically through multiple engine combinations of pulse positions adjustment control. aircraft scheduled landing spot for 19.51W,44.12N, elevation $-2641 \mathrm{~m}$.

aircraft in a predetermined region of accurate soft landing, landing is the key track and control strategy of design. Landing trajectory design of the basic requirements are: track point $15 \mathrm{~km}$ in recent months, far-month point $100 \mathrm{~km}$ elliptical orbit; landing track in recent months, to the landing site, its descent is divided into 6 stages, optimize the 6 stages and error analysis of control strategies, is the key to this model. 


\section{2 problem}

(1) determine the landing preparations track points and far-month point position in recent months, as well as aircraft speed and direction.

(2) determine the moon landing of the third rail and optimal control strategy in 6 stages.

(3) for designing the landing track and control tactics of error analysis and sensitivity analysis.

\section{Problem analysis}

Considered actual flight situation, established landing cabin mathematics model description aircraft, of movement State, according to established of model, in MATLAB environment in the on bent over elevation for repeatedly set, looking for meet design requirements optimal solutions, obtained optimal design points location of cross, and ordinate; in according to geometry obtained in recent months points and optimal design points of angle, according to longitude degrees calculation method, determines in recent months points, and far months points of location; Dang aircraft, When the aircraft landing elliptical orbit, he follows the conservation of energy, so aircraft, in recent months points and far months points of energy equal, and due to aircraft, in in recent months points and far months points of speed are and aircraft, to months heart of line vertical, is angular momentum conservation, according to gold proportions substitution, obtained has in recent months points and far months points of speed size and direction.

\section{The model assumptions}

According to the actual situation and the needs of model simplification, solving, this article makes the following assumptions:

(1)that the Moon is ideal sphere, regardless of the Non-spherical gravitational field of the Moon and Lunar effects resulting from the flat rate;

(2) Assuming the slowdown strictly track landing of aircraft in flight, the pitch angle set to small, the lander yaw;
(3) aircraft $100 \mathrm{~km}$ circular orbits and elliptical orbits are in the composition of the lunar surface and Meridian circles on the same plane.

(4)during the process of landing, aircraft considers only the gravitational force of the Moon, other celestial bodies is not to be considered (such as the Earth, the Sun), gravity, and other external factors.

(5) slow deceleration phase, aircraft constant deceleration of linear motion.

\section{Establishing and solving of the model}

4. 1 Determine the position in recent months point farthest from the moon

(1) model - model Lunar Lander of Lander is a strongly nonlinear systems.

While you're designing displays guidance law for, there is a need in some reasonable assumptions on the basis of simplified them. This ignores the Moon's rotation, and that the Moon is a good ball, regardless of the Non-spherical gravitational field of the Moon and Lunar effects resulting from the flat rate, and does not take into account quality of Lander's changes.

Lander model coordinate system used in a coordinate system, brake and body coordinate systems. The origin of the coordinate system is located in the heart month; braking moments where the lander mass of $\mathrm{x}$ axis pointing to the intersection of Meridian and Equator; $\mathrm{z}$ axis perpendicular to the plane of the equator, pointing to the Moon's North Pole; y-axis constitutes a right-handed Cartesian coordinate system. Brake origin located in Lander mass month in the soft landing braking moment heart vector and the intersection of the Moon's surface; y axis along the lander mass origin and the brake moment connection direction; $\mathrm{x}$ axis at the landing during the orbital plane of the moon lander runs the brake, with the $y$ axis, and point to the Lander's direction; $\mathrm{z}$-axis constitutes a right-handed coordinate system. Coordinate diagram as shown in Figure 1: 


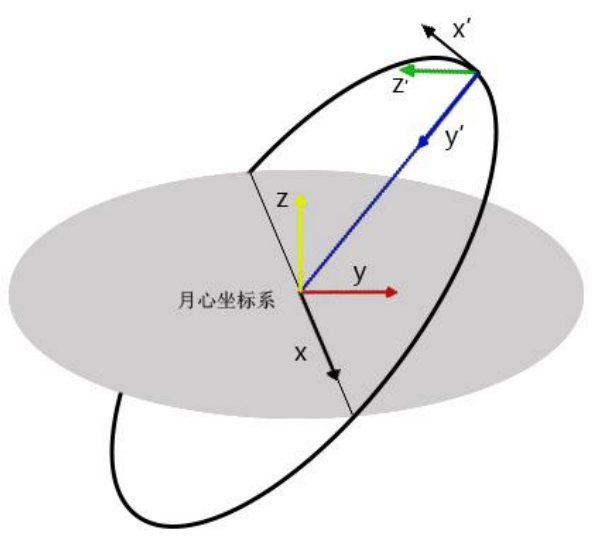

Figure 1 coordinate system diagram

Here's what Lander model ${ }^{[1]}$

$$
\left\{\begin{array}{l}
\frac{d v_{x}}{d t}=\frac{P}{m} \cos \varphi \cos \psi \\
\frac{d v_{y}}{d t}=\frac{P}{m} \sin \varphi \cos \psi-\frac{f M}{r^{2}} \\
\frac{d v_{z}}{d t}=-\frac{P}{m} \sin \psi \\
\frac{d x^{\prime}}{d t}=v_{x} \\
\frac{d y^{\prime}}{d t}=v_{y} \\
\frac{d z^{\prime}}{d t}=v_{z}
\end{array}\right.
$$

In the above formula, $P$ is the thrust, $\varphi_{\text {is }}$ pitch angle, the gravitational constant $\mu=f M=4.9028 * 10^{10}\left(\mathrm{~m}^{3} / \mathrm{s}^{2}\right), \psi$ for the yaw angle, $r$ distance to Lander's Center of mass to the months.
(2) The solution of the model

According to the model, solution of ordinary differential equations using MATLAB, which also requires the use of a given initial value for iterative method of calculation, get the optimal volume of a set of optimal control. Calculation too complex and therefore made the following analysis.

Considered actual flight situation, in main deceleration segment of level displacement maximum, In the main reduction period of horizontal, horizontal displacement is the greatest, so solution and target points of distance location in the we only considered main deceleration segment flight of distance; due to Moon of average RADIUS for $1737.013 \mathrm{~km}$, and brake points (that in recent months points) away from Moon surface of distance for $15 \mathrm{~km}$, difference larger, so pitching angle $\varphi$ set for small; assumed deceleration in the aircraft strictly along landing track, Lander yaw Angle is $\psi \approx 180^{\circ}$

In MATLAB environment in the on bent over elevation for repeatedly set, looking for optimal solutions (code see Appendix a), have aircraft, away from months surface distance with time changes figure, as Figure 2 by shows, in 516 seconds Shi, aircraft, reached away from months surface $3 \mathrm{~km}$ of design requirements, completed main deceleration segment; $x$ axis direction Shang displacement with time changes figure, as Figure 2, have 516 seconds Shi x direction Shang displacement $653 \mathrm{~km}$. 


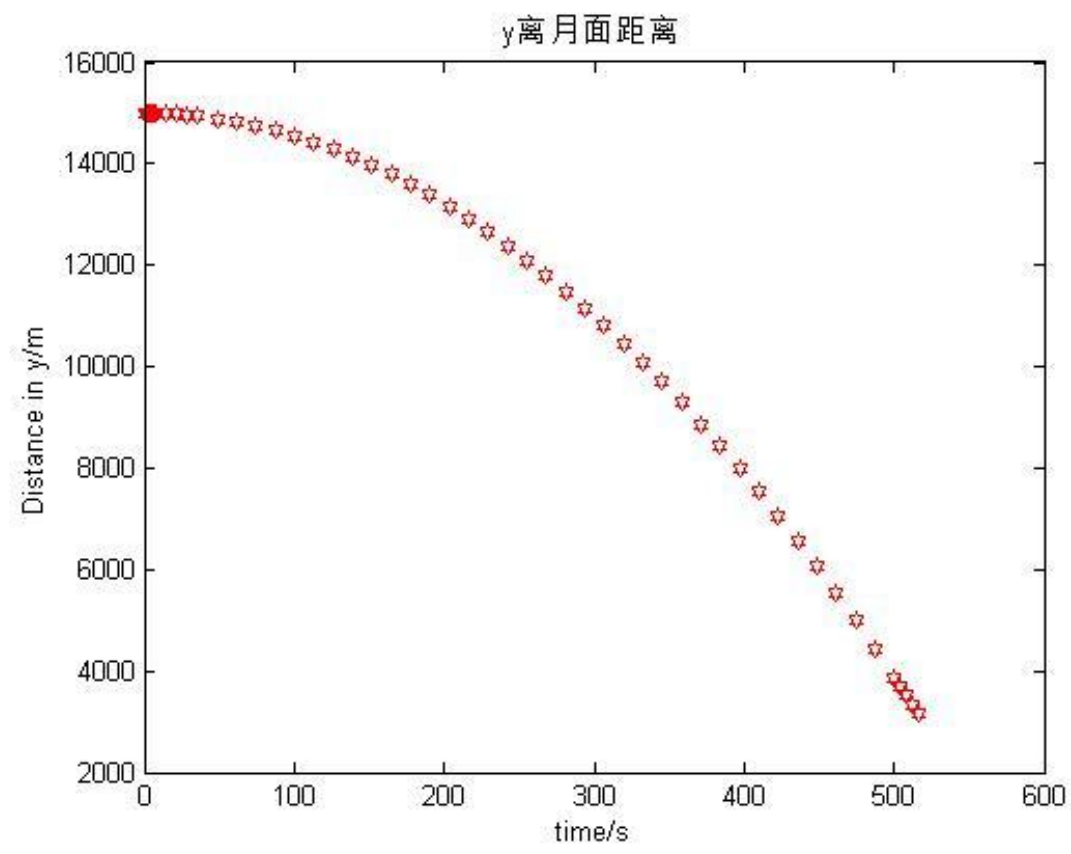

Figure $2 \mathrm{x}$ axis displacement

According to geometry have in recent months points and main deceleration end (away from months surface $3 \mathrm{~km}$ ) of angle for, due to aircraft, of landing points for $\left(19.51^{\circ} \mathrm{W}, 44.12^{\circ} \mathrm{N}\right)$, and in main deceleration end and landing points stage myopia line movement, is deceleration end and landing points of longitude degrees same, and by longitude degrees of calculation method have in recent months points of longitude degrees $\left(19.51^{\circ} \mathrm{W}, 22.52^{0} \mathrm{~N}\right)$, far months points of longitude degrees $\left(160.49^{\circ} \mathrm{E}, 22.52^{\circ} S\right)$.

\subsection{To determine the magnitude and direction of velocity}

(1) The establishment of the model

When the moon landing ellipse orbit ready at third, to represent points in recent months, and months of little speed, according to energy conservation, aircraft equivalence point and long months of energy in recent months, so have

$$
\frac{1}{2} M V_{1}^{2}-\frac{G M m}{R_{1}}=\frac{1}{2} M V_{2}^{2}-\frac{G M m}{R_{2}}
$$

$M \mathrm{M}$ is the mass of the Earth, $G$ is the gravitational constant, $R_{1}$ and $R_{2}$ is in recent months and far-month to month in the distance.

aircraft in recent months and months little of speed aircraft perpendicular to the line of the month, according to the conservation of angular momentum:

$$
M V_{1} R_{1}=M V_{2} R_{2}
$$

Notes that the golden ratio replacement:

$$
G M=g R^{2}
$$

From a (1), (2), (3), we can get

$$
V_{1}=\sqrt{\frac{R_{2}}{R_{1}} \frac{2 g}{R_{1}+R_{2}}} R \quad V_{2}=\sqrt{\frac{R_{1}}{R_{2}} \frac{2 g}{R_{1}+R_{2}}} R
$$


(2) the model solution

According to the data:

$$
R_{1}=R+H_{1}
$$

$R_{2}=R+H_{2}$

$H_{1}, H_{2}$ as in recent months and far months points to the distance of the Moon's surface,

Based on MATLAB programming and related data into (as seen in Appendix II):

$$
V_{1}=1.7144 \mathrm{~km} / \mathrm{s}, V_{2}=1.6351 \mathrm{~km} / \mathrm{s}
$$

By looking in the literature [2] showed that the Moon's escape velocity $v_{\text {逃 }}=2380 \mathrm{~m} / \mathrm{s}$, apparently obtained in recent months and months of little's velocity is more accurate.

Analysis of aircraft landing ellipse ready to track the physical properties of trajectory analysis diagram is shown below:

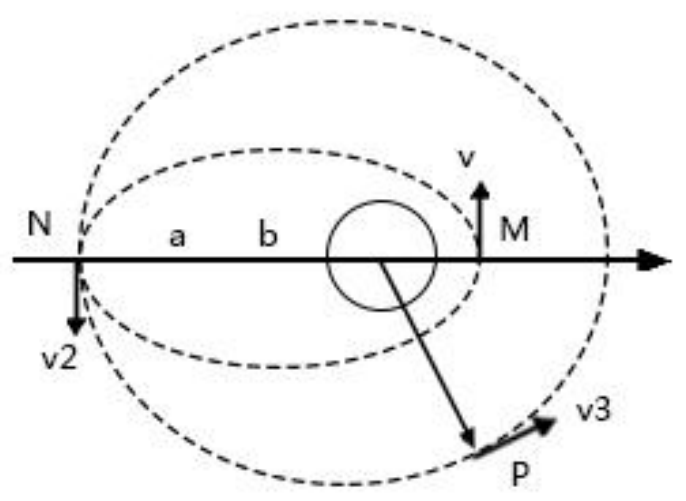

Figure 3 schematic diagram of distant perihelion

Figure 3 shows that aircraft in recent months and months little velocity perpendicular to the connection with the goddess the third month, that is prepared for landing ellipse tangent to the orbit direction.

\subsection{Determine the landing trajectory}

(1)The establishment of the model aircraft in landing rail stage of time more short, General in hundreds of seconds of range within, also, due to Moon surface near no atmospheric, so in by force analysis in the no atmospheric resistance of effects, Moon of gravitational only Earth gravitational of one-sixth around, since corner speed also only Earth angle speed of one-twenty Nineth around, so can ignored by Moon rotation caused brother type force and traction of effects, in above assumed Xia, we according to Newton second law: F=Ma, Kinematic differential and aircraft stress condition analysis, establish landing dynamic model group:

$$
\left\{\begin{array}{l}
\frac{d v_{x}}{d t}=-\frac{v_{e} \dot{m}}{m_{o}-m t} \cos (\theta+\beta) \\
\frac{d v_{y}}{d t}=-\frac{v_{e} \dot{m}}{m_{o}-m t} \sin (\theta+\beta)+g_{\text {月 }} \\
\tan \beta=\frac{v_{y}}{v_{x}}
\end{array}\right.
$$

Type: $v_{x}$ for the velocity along the $\mathrm{x}$-direction, $v_{y}$ for the velocity along the y-direction; $\theta$ is the angle between the direction of the thrust direction and speed, $\beta$ is the angle between velocity and direction of water level, $\dot{m}$ is the number of kilograms of fuel consumption per unit time, $m_{o}$ is the goddess of initial landing point of the third quality; $m_{t}$ for aircraft in landing the quality at any time in the process. $g_{\text {月 }}$ for Lunar gravity 
(2) The solution of the model

The relationship between speed and time are calculated using MATLAB programming (as seen in Appendix III).

$$
V_{x}=1730.6-3.7872 * \mathrm{t}
$$

$V_{y}=1.2336 * t+0.0016994 * t^{2}-9.6301 * 10^{-6} * t^{3}$

$$
+1.4389
$$

The above equations are integral relationship between displacement and time:

$S_{x}=-1.8936 * t^{2}-1730.6 * t$

$S_{y}=-2.4075 * 10^{-6} * t^{4}+5.6647 * 10^{-4} * t^{3}+\frac{771}{1250} * t^{2}+1.4389 * t+15000$

Displacement to the $\mathrm{x}$ and $\mathrm{y}$ direction to merge aircraft final equation:

$Y=15024.0=1.656 * x^{2}-6.2058 * 10^{-13} * x^{3}-0.002975 * x$

(3)Simulation of model test

According to aircraft final trajectory equation trajectory simulation using MATLAB Figure 4 below:

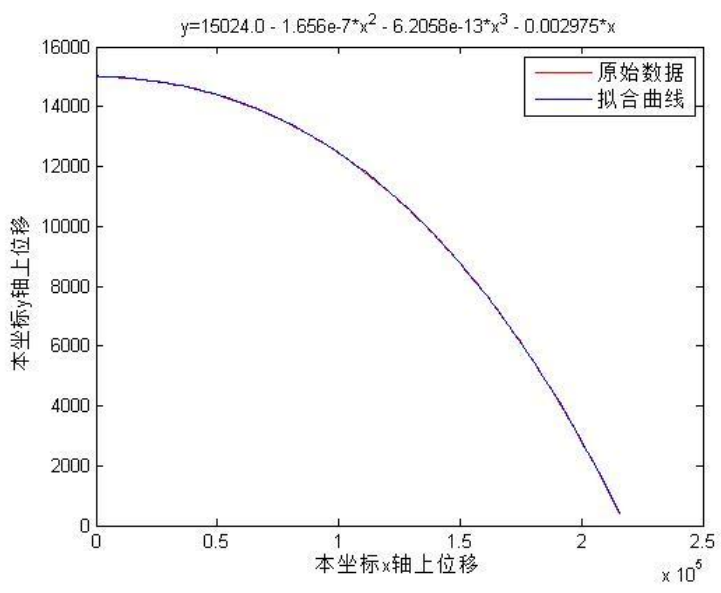

Figure 4 orbit equation

From know aircraft on the origin trajectory curve by curve fitting the model with us much closer to trend is basically the same, and thus, checked them out to establish aircraft final trajectory equation for accuracy.

\section{CONCLUSIONS}

In this paper, in determining the size of the speed, the use of the law of conservation of energy and angular momentum conservation law, easy to understand, gradually in-depth. By analyzing the movement of the aircraft and stress, we according to the Newton's second law: $F=m a$, the kinematic differential method and the stress points, aircraft soft landing dynamic equation model group, for the mathematical process of solving the model is simple, clear physical concept, and combined with the geometric mapping, illustrated, more persuasive; More evident on the model test and simulation, the accuracy of the model. Established model and the actual close relation, it is effective to use the simple model, have very good generality and extension. Using Matlab and EXCELL software to calculate, high credibility.

\section{References}

[1] Chang Xiaofei, Fu Wenxing, Yanjie. Lunar Lander braking deceleration control $[\mathrm{J}]$. School of Northwestern Polytechnical University aerospace. 2008,26 (5): 627 630

[2] http://zh.Wikipedia.org/wiki/to the moon

[3] the Qiu Shi science and technology. From entry to the master MATLAB7.0. PT press. 2006

[4] Jiang qiyuan, Xie Jinxing, ye Jun. mathematical model. higher education press, 2005. 